\title{
Mindfulness: reflexão sobre limites e potencialidades para a assistência de enfermagem
}

Mindfulness: critical thinking on limits and potentialities for nursing assistance

Mindfulness: reflexión sobre límites y potencialidades para la asistencia de enfermería

\section{Edilaine Cristina da Silva Gherardi-Donato', Maria Neyrian de Fátima Fernandes ${ }^{\text {II }}$ Fabio Scorsolini-Comin ${ }^{\text {III }}$, Ana Carolina Guidorizzi Zanetti ${ }^{\text {IV }}$}

\begin{abstract}
Resumo: Objetivo: refletir sobre os limites e as potencialidades de mindfulness para a assistência de enfermagem, explorando as evidências científicas disponíveis, o uso na assistência à saúde e na assistência de enfermagem. Método: a reflexão pautou-se nos tópicos: Mindfulness e evidências científicas, Mindfulness na assistência à saúde, e Mindfulness na assistência de enfermagem. Resultados: o potencial das práticas de mindfulness foi evidenciado para tratar e prevenir problemas relacionados com o estresse e promover estratégias de enfrentamento consideradas mais assertivas, tanto para os clientes como para os próprios profissionais de enfermagem. As limitações envolveram a insipiência da temática no âmbito da enfermagem brasileira e no delineamento dos estudos, pois as intervenções baseadas em mindfulness requerem protocolos mais longos que exigem capacitação e engajamento na prática. Considerações finais: evidências apontam diversas potencialidades de intervenções baseadas em mindfulness para o autocuidado e atenção aos usuários e familiares, promovendo assistência integral e prescrevendo uma perspectiva inovadora para o cuidado.
\end{abstract}

Descritores: Enfermagem; Atenção plena; Assistência integral à saúde; Práticas integrativas

\footnotetext{
IEnfermeira, Doutora em Enfermagem Psiquiátrica, Professora Associada EERP-USP, Ribeirão Preto, São Paulo, Brasil. E-mail: gherardidonato@gmail.com ORCID ID: https://orcid.org/0000-0001-7475-6650

IIEnfermeira, Doutora em Enfermagem Psiquiátrica, Professora Adjunta UFMA, Imperatriz, Maranhão, Brasil. Email:neyrianfernandes@gmail.com ORCID ID: https://orcid.org/0000-0001-7626-9733

IIIPsicólogo, Doutor em Psicologia, Professor Doutor EERP-USP, Ribeirão Preto, São Paulo, Brasil. E-mail:fabio.scorsolini@usp.br ORCID ID: https://orcid.org/0000-0001-6281-3371

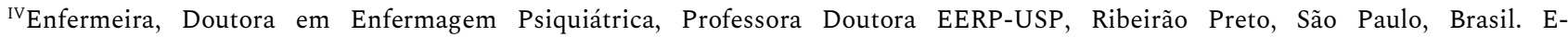
mail:carolzanettieerp@gmail.com ORCID ID: https://orcid.org/0000-0003-0011-4510
} 


\begin{abstract}
Aim: to think critically about the limits and potentialities of mindfulness for nursing care, exploring available scientific evidence, its use in health care and nursing care. Method: the critical thinking based itself on the topics: Mindfulness and scientific evidence, Mindfulness in health care, and Mindfulness in nursing care. Results: the potential of mindfulness interventions was evidenced to treat and prevent stressrelated problems and to promote coping strategies considered more assertive, both for clients and for the nursing professionals themselves. The limitations involved the insipidity of the subject in the scope of Brazilian nursing and in the design of the studies, since interventions based on mindfulness require longer protocols that require training and engagement in the practice. Final considerations: evidence syndicate several potentialities of mindfulness based interventions for self-care and attention to users and families, promoting comprehensive care and prescribing an innovative perspective for care.
\end{abstract}

Descriptors: Nursing; Mindfulness; Comprehensive health care; Integrative practices

Resumen: Objetivo: reflexionar sobre los límites y las potencialidades del mindfulness para la asistencia de enfermería, explorando las evidencias científicas disponibles, el uso en la asistencia a la salud y en la asistencia de enfermería. Método: la reflexión se basó en los temas: Mindfulness y las evidencias científicas, Mindfulness en la asistencia a la salud, y Mindfulness en la asistencia de enfermería. Resultados: el potencial de la práctica de mindfulness fue evidenciado para tratar y prevenir problemas relacionados alestrés y para promover estrategias de enfrentamiento consideradas más asertivas, tanto para los clientes cuanto para los propios profesionales de enfermería. Las limitaciones se relacionaron a la insipiencia de la temática en el ámbito de la enfermería brasileña y en el delineamiento de los estudios, pues las intervenciones basadas en mindfulness requieren protocolos más largos que exigen capacitación y compromiso en la práctica. Consideraciones finales: evidencias revelan diversas potencialidades de las intervenciones basadas en mindfulness para el autocuidado y atención a los usuarios y familiares, promoviendo asistencia integral y prescribiendo una perspectiva innovadora para el cuidado.

Descriptores: Enfermería; Atención plena; Asistencia integral a la salud; Prácticas integradoras

\title{
Introdução
}

Mindfulness ou atenção plena é um estado de consciência plena promovido a partir do escolha consistente e deliberada do indivíduo para tomar conhecimento do que está ocorrendo nos mundos interno e externo, exercitando a capacidade de estar completamente envolvido no momento presente, sem desviar sua atenção com eventos do passado ou do futuro. ${ }^{1-2}$ É a consciência que surge quando se presta atenção de uma maneira específica, ou seja, com propósito de perceber o momento presente sem julgamentos. ${ }^{2}$

Embora possa ocorrer diferenças individuais no grau natural de atenção plena entre as pessoas, esse tipo de habilidade metacognitva pode ser desenvolvida com treino. ${ }^{1}$ Tais 
técnicas visam a desenvolver uma perspectiva para os pensamentos e sentimentos nos quais estes sejam reconhecidos como eventos mentais ou como reflexos precisos da realidade. $^{2}$

Comumente, as pessoas e os críticos tendem a associar mindfulness à prática de meditação. Contudo, essa prática reúne uma variedade de métodos, tais como yoga, respiração focada, consciência corporal e meditação. Estudos ampliam as possibilidades de praticar mindfulness, que seria por meio das artes, da literatura e até mesmo por meio de atividades do cotidiano. Todas essas práticas têm origens e efeitos fisiológicos e são empregadas para reforçar a consciência e buscar o bem-estar. ${ }^{1-2}$

No cuidado em saúde, a prática de mindfulness ajuda a cultivar no profissional um desejo de saber e entender os sentimentos e necessidades de pacientes e familiares, desenvolvendo a capacidade de estar totalmente presente com a atenção focada no outro, paciente e/ou família. ${ }^{2}$ A "presença” do enfermeiro é um elemento crítico para um apoio efetivo ao paciente que experimenta algum desequilíbrio físico ou mental. Atuando como assistencialistas, professores e pesquisadores, os enfermeiros podem lançar mão de técnicas voltadas ao aprendizado da atenção plena para engajar-se ativamente nos acontecimentos que ocorrem nos contextos ao seu redor. ${ }^{2}$

Nesse sentido, diferentes abordagens de programas de intervenção em mindfulness têm sido empregadas nesses diversos contextos. Dentre eles, destaca-se o Programa Mindfulness para Redução do Estresse $(\mathrm{MBSR})^{3}$ de oito semanas, que reúne variadas técnicas de meditação para promover a qualidade mindfulness, com o objetivo de ensinar as pessoas a abordarem situações estressantes conscientemente para que elas possam responder assertivamente ao invés de reagir automaticamente. A realização de práticas de redução de estresse baseadas em mindfulness vem apresentando resultados positivos promissores em diversas populações clínicas, nas quais a enfermagem é apontada como 
Mindfulness: reflexão sobre limites e potencialidades para a assistência de enfermagem | 4

uma das profissões de maior protagonismo, justificada pela sua posição de destaque na gestão e no oferecimento dos cuidados de saúde in loco.

De acordo com a intenção das tessituras apresentadas, questiona-se: quais seriam os limites e as potencialidade de mindfulness para a assistência de enfermagem? Percebe-se que refletir sobre mindfulness é importante por mostrar um campo fértil de exploração para a enfermagem, fornecendo fundamentos consistentes para aproximação das suas práticas como um instrumento para a melhoria do cuidado e a efetivação de uma assistência integral. Assim, objetiva-se refletir sobre os limites e as potencialidades das práticas de mindfulness para a assistência de enfermagem, explorando as evidências científicas disponíveis, o uso na assistência à saúde e na assistência de enfermagem.

\section{Mindfulness e evidências científicas}

Para compreender como a prática de mindfulness afeta indivíduos, grupos e instituições, um conjunto consistente de estudiosos têm se dedicado a descrever os efeitos deste estado de mente sobre funções humanas básicas que incluem atenção, cognição, emoção, comportamento e fisiologia. ${ }^{4}$ Os impactos do cultivo de mindfulness sobre a atenção reportam uma melhor estabilidade (sustentação da atenção em algo presente e uma mente menos vaga), melhor controle da atenção (capacidade de focar em algo selecionado), e melhor eficiência de atenção (uso econômico dos recursos cognitivos). ${ }^{4}$

A melhoria na qualidade da atenção representa provimento de uma melhor performance cognitiva em dois principais aspectos: capacidade e flexibilidade. A capacidade cognitiva foi considerada em relação à memória e fluidez da inteligência a partir do desempenho no armazenamento e processamento de experiências e informações. A progressão na flexibilidade cognitiva remete à melhoria na capacidade de adaptação através da criação de novas respostas e perspectivas para as situações apresentadas, associando mindfulness à criatividade e ao pensamento convergente e divergente. ${ }^{5}$ 
Quanto às emoções, os estudos revelam o impacto sobre a reatividade e o tom emocional. Através da atenção qualificada, o cultivo de mindfulness pode influenciar na seleção dos estímulos emocionais para observação e alterar como eles são avaliados, moldando as reações emocionais. A prática está associada a tons emocionais menos negativos e mais positivos, acelerando a recuperação das emoções negativas e encurtando o tempo de reação emocional diante de uma situação adversa. ${ }^{6}$

Em termos comportamentais, destaca-se a redução da automaticidade, pois permite maior conscientização das operações automáticas e habituais, as quais ocorrem quando há envolvimento em comportamentos sem esforço, sem a supervisão consciente de seus detalhes operacionais. Ainda promove ativação em regiões mais elevadas do cérebro associadas ao autocontrole comportamental, aumentando a possibilidade do indivíduo de reconhecer a ocorrência de comportamentos automáticos, cujas respostas podem ser pouco adaptativas, tais como as compulsões e dependências em geral. ${ }^{6-7}$

$\mathrm{Na}$ perspectiva fisiológica, as evidências científicas produzidas têm enfatizado o papel do cultivo de mindfulness no estresse, mostrando que a prática está relacionada com diversos mecanismos neurobiológicos responsáveis pela regulação do estresse. Entre os achados, pontua-se seus efeitos benéficos sobre o cortisol, a qualidade do sono, o processo de envelhecimento, o sistema imunológico, os processos inflamatórios e a estrutura do tecido cerebral. ${ }^{4}$

Evidências mostram mudanças nas propriedades funcionais cerebrais após a prática de mindfulness. Imagens de Ressonância Magnética (IRM) que investigaram a neuroplasticidade em regiões que regulam o controle da atenção, mostraram efeitos maiores no córtex cingulado anterior, responsável pela atenção executiva, flexibilidade para mudança de estratégias e controle ao perceber a presença de conflitos emergentes. ${ }^{2}$ Lesões nessa região levam à impulsividade e agressão descontrolada; o comprometimento da 
Mindfulness: reflexão sobre limites e potencialidades para a assistência de enfermagem I 6

conexão do córtex cingulado anterior com outras regiões do cérebro acarreta o baixo desempenho da flexibilidade mental, dificultando o processo de tomada de decisão. ${ }^{8}$

Até o momento, foram identificadas oito regiões cerebrais consistentemente alteradas em grupos de meditadores, além do córtex cingulado anterior, como o córtex fronto-polar, responsável pela metaconsciência; o córtex sensorial e a ínsula onde ocorre a consciência corporal das sensações exteroceptivas e interoceptivas; o aumento da massa cinzenta no hipocampo, base para a consolidação e reconsolidação da memória e controle do estresse, pois é uma região coberta por receptores do cortisol; o cingulado anterior e médio, e o córtex orbitofrontal que regulam as emoções e o ego; o fascículo longitudinal superior e o corpo caloso que regulam a comunicação intra e inter-hemisférica. ${ }^{8-9}$

Essas evidências mostram que a prática de mindfulness afeta áreas cerebrais importantes para o conhecimento de si e dos outros. Os resultados encontrados até o momento são desafiadores e estimulantes, o que torna lícito afirmar que tal prática compõe uma ferramenta de intervenção importante para a assistência à saúde.

\section{Mindfulness na assistência à saúde}

Diversos profissionais da saúde têm utilizado intervenções baseadas em mindfulness em diferentes condições físicas e psíquicas nos contextos clínicos e terapêuticos. Em busca de uma assistência integral, é necessário desenvolver uma compreensão da prática de mindfulness na sua interface com diversos níveis de conhecimento para avaliar de forma adequada seus efeitos. Sua utilização vem se fortalecendo com o uso crescente de práticas integrativas e complementares não farmacológicas para manejo de condições clínicas agudas e crônicas influenciadas pelo estresse e outros problemas de saúde, reportando os benefícios dessas práticas. ${ }^{3}$

Há fortes evidências científicas de que as intervenções baseadas em mindfulness podem melhorar uma série de resultados biológicos e psicológicos em doenças, incluindo 
dor aguda e crônica, hipertensão, diabetes tipo 2, Síndrome da Imunodeficiência Adquirida/Vírus da Imunodeficiência Humana (HIV/AIDS), depressão e abuso de drogas. ${ }^{10}$ Tais condições são indiscutivelmente presentes no cotidiano de trabalho da enfermagem e exigem intervenções para a efetividade do cuidado integral.

O MBSR, um dos programas mais investigados na literatura científica, é composto pelas práticas de meditação formal e informal. A formal estimula a atenção focada na respiração, na varredura corporal, no monitoramento aberto da experiência no presente momento sem julgamento e durante as atividades de caminhar e comer. A informal é realizada durante atividades do cotidiano, o que consiste em viver essas atividades momento a momento com plenitude e engajamento. ${ }^{2,11}$

Esse programa aprimora a habilidade de observar o conteúdo dos eventos imediatos, mais especificamente a natureza transitória dos pensamentos, emoções, memórias, imagens mentais e sensações físicas. Diminui a tendência habitual de reagir emocionalmente e ruminar os pensamentos transitórios ou as sensações físicas, reduzindo os sintomas de depressão e ansiedade, fortalecendo as funções imunológicas, o autocontrole emocional, a orientação volitiva da atenção e, sobretudo, o equilíbrio necessário para lidar com os estressores. ${ }^{12}$

Entre os impactos mais preocupantes do estresse, considera-se, além dos prejuízos diretos à saúde e à qualidade de vida, o comprometimento das habilidades e competência para o trabalho, e a adoção de comportamentos não adaptativos como uso de drogas psicoativas. Por isso, considera-se relevante a busca por uma intervenção de redução de estresse que objetive evitar adoecimentos pelo estresse contínuo, especialmente entre os grupos que apresentam alta incidência de estresse laboral e problemas de saúde fortemente influenciado pela experiência de estresse, como enfermeiros, pessoas expostas à violência e usuários de drogas. 
Mindfulness: reflexão sobre limites e potencialidades para a assistência de enfermagem I 8

No escopo de cuidados ao uso de drogas e à dependência química, o Programa de Prevenção de Recaída Baseada em Mindfulness (MBRP) procura identificar as situações de alto risco desencadeadoras de recaídas em indivíduos com comportamento aditivo. As evidências quanto à integração de práticas de mindfulness com a prevenção tradicional de recaída cognitivo-comportamental sugerem a possibilidade de desenvolvimento de uma relação de conscientização e não identificação com pensamentos e sentimentos, evitando o surgimento de padrões de pensamento e comportamentos que podem levar à recaída. ${ }^{13}$

Por meio da conscientização, regulação e tolerância dos potenciais gatilhos de recaída, o cultivo de mindfulness ajuda a manter a capacidade de lidar com os fatores precipitantes da recaída, quebrando o ciclo do comportamento automático de uso de substâncias psicoativas. Contribui para a conscientização e aceitação em casos de lapsos nesse processo, ajudando a pessoa a reconhecer e minimizar o sentimento de culpa, o que também diminui o risco de recaída. ${ }^{13} \mathrm{O}$ programa mostrou eficácia na redução de $31 \%$ no uso de álcool e outras drogas em um período de até um ano após a intervenção. ${ }^{4}$

Para a prevenção das recaídas de depressão, o programa de Terapia Cognitiva baseada em Mindfulness (MBCT) mostrou-se eficaz, contribuindo para a resolução dos sintomas residuais em pessoas com depressão recorrente, pois seu diferencial em relação às outras terapias consiste na incorporação das habilidades de mindfulness no cotidiano. ${ }^{14}$ Dessa forma, o cultivo de mindfulness mostrou-se tão eficaz quanto o antidepressivo na prevenção das recaídas.

Entre usuários de um serviço de saúde submetidos a programas de relaxamento baseados em mindfulness e yoga, ${ }^{10}$ houve uma diminuição de $43 \%$ da utilização dos serviços quando comparados com o ano anterior, mostrando que esse tipo de intervenção pode representar uma economia em saúde. Assim, o foco da assistência à saúde tenderá a mudar da super utilização da clínica especializada no tratamento de usuários em estágios 
avançados da doença para uma abordagem mais centrada no indivíduo e um cuidado coordenado por equipes que promovam o bem-estar, o autocuidado, a prevenção e o manejo da doença.

Com base nas evidências sobre os programas de intervenção, os praticantes mostraram melhora significativa nos sintomas clínicos, na ansiedade e na qualidade de vida, compondo uma estratégia disponível que contribui para a saúde mental dos indivíduos. Dessa forma, pode-se atuar com maior eficiência à medida que suas ações estejam sustentadas por um modelo de cuidado integral que reconheça os estados de saúde e doença como complexos e multicausais.

\section{Mindfulness na assistência de enfermagem}

O envolvimento da enfermagem com medidas terapêuticas não farmacológicas sustentadas por fortes evidências científicas ampliam as possibilidades para a atuação do enfermeiro e a efetividade das ações de promoção e prevenção em saúde. ${ }^{11}$ Neste sentido, a utilização dessas medidas contribui para evitar adoecimentos decorrentes do estresse contínuo, à medida que o enfermeiro orienta seus clientes a reconhecerem a resposta negativa do corpo ao estresse prolongado e fornece um recurso para que eles possam interromper o impacto negativo dessa resposta.

A utilização de intervenções de redução de estresse baseadas em mindfulness tem sido investigada e discutida como uma ferramenta clínica não farmacológica adequada, cuja inclusão na prática de enfermeiros deflagra a necessidade de produção de conhecimento a partir de estudos que investiguem sua efetividade na perspectiva da pesquisa em enfermagem.

As possibilidades do mindfulness podem ser ampliadas inclusive para o autocuidado, pois o próprio profissional de enfermagem está submetido aos riscos relacionados ao estresse. Sabe-se que as responsabilidades atribuídas à enfermagem são 
situações de tensão diversas. Adicionalmente, desfechos como ansiedade, depressão e dores musculares encontram-se associados ao estresse vivenciado por profissionais da equipe de enfermagem, na qual a chance de desenvolver esses agravos chega ao dobro em profissionais que apresentam alto nível de estresse..$^{15-16}$

Embora possa ser ensinado e aperfeiçoado por meio de treinamentos, a prática de mindfulness é desafiadora em termos de manutenção e fortalecimento, principalmente diante de estímulos árduos e estressores. A prática constante resulta em compaixão consigo mesmo e os outros, pois quando o praticante torna-se mais habituado a aceitar o momento como ele é, sem julgamento, constrói-se um retrato mais realista dos fatos, contribuindo na melhora da tomada de decisão ou na comunicação interpessoal.

A atenção plena, a consciência do momento presente e a compaixão estão interligadas e são elementos importantes na interação com o sofrimento do outro. A empatia é importante nesse processo por estabelecer uma ponte que pode ser atravessada até o território dos sentimentos de outro indivíduo, mas pode-se dizer que a compaixão é ainda mais necessária, pois entende-se que a tendência humana é ser empático apenas com os seus conhecidos ou semelhantes. No sistema de cuidado, a compaixão vai além, pois ela não se limita aos conhecidos e é menos estressante do que a empatia. ${ }^{17}$

O treino da compaixão por meio da meditação tem tornado o praticante mais propenso a ajudar o outro, isso com apenas dois dias de treino. Já a prática constante pode induzir uma mudança duradoura da motivação para a compaixão, independente da pessoa ou situação. Assim, percebe-se que o treinamento da compaixão exerce uma influência importante na ajuda altruística, sendo relevante implementá-lo no cuidado em enfermagem. ${ }^{18}$

Nesse contexto, a Enfermagem pode beneficiar-se da compaixão, especialmente pela sua relação de proximidade com os usuários, cujas ações incluem o reconhecimento das situações geradoras de estresse, a detecção de estratégias de enfrentamento e a capacidade 
para intervir adequadamente na redução dos agentes e dos efeitos do estresse nos indivíduos e também na coletividade. ${ }^{19}$

A falta de compaixão no cuidado em enfermagem foi notícia na Inglaterra quando uma associação de pacientes denunciou a negligência desses profissionais em instituições de longa permanência para idosos. De imediato, o governo exigiu que as universidades implementassem padrões mais elevados de formação nos cursos de enfermagem. Algumas universidades alegaram redução nas verbas destinadas à formação de enfermeiros, mas as discussões discorreram sobre a qualidade da formação e a seleção profissional para os serviços de saúde. Declarou-se que a qualificação estava pouco voltada para as necessidades dos usuários, afastando os enfermeiros do desenvolvimento de componentes interpessoais básicos e essenciais. ${ }^{20-21}$

Para minimizar problemas dessa natureza, a prática de mindfulness tem sido incorporada na formação profissional de médicos e enfermeiros. Nos Estados Unidos, cerca de $79 \%$ dos cursos de medicina oferecem algum tipo de treinamento em mindfulness, bem como centros de pesquisa e formação em mindfulness tem proliferado mundialmente. ${ }^{22} \mathrm{O}$ treinamento em estudantes de enfermagem mostrou que houve melhora em alguns aspectos da tomada ética de decisão. Entretanto, mesmo com todas as evidências dos benefícios da técnica, ainda existem poucos cursos de enfermagem que utilizam esse tipo de treinamento nos seus currículos. ${ }^{23}$

No Brasil, com o intuito de cuidar e promover a saúde além das práticas tradicionais foi fundado em 2011 o Centro Mente aberta da Universidade Federal de São Paulo, na cidade de Santo Amaro, com atuação na prática profissional baseados em Mindfulness. Em 2016, na Universidade de São Paulo, foi inaugurado o Centro de Mindfulness e Terapias Integrativas junto à Escola de Enfermagem de Ribeirão Preto (EERP-USP), coordenado por professoras de enfermagem e pesquisadoras na área de saúde mental. Cabe destacar a 
existência de outros centros de disseminação das práticas de mindfulness recentes e até mais antigos de natureza não acadêmica.

O Centro de Mindfulness e Terapias Integrativas da EERP-USP, constituído no contexto acadêmico e de pesquisa em enfermagem de saúde mental, integra atividades de pesquisa, ensino e extensão, buscando alinhamento com a Política Nacional de Práticas Integrativas e Complementares em Saúde (PNPIC) aprovada pelo Ministério da Saúde brasileiro por meio da Portaria n 633 , de 28/03/2017 e pela Portaria nำ 702, de 21/03/2018, cujos objetivos envolvem medidas como a incorporação e implementação de práticas integrativas no Sistema Único de Saúde (SUS) em todos os níveis de atenção à saúde, aumenta a resolubilidade do SUS por meio da ampliação do acesso às práticas integrativas, promove ações de saúde utilizando alternativas inovadoras e contribuidoras para o desenvolvimento sustentável de comunidades. ${ }^{24-26}$

As práticas integrativas contempladas pela PNPIC, iniciada em 2006 com cinco práticas reconhecidas, 14 em 2017 e 10 em 2018, totalizam um conjunto de 29 práticas, entre as quais a meditação. Em Ribeirão Preto, São Paulo, ocorreu a aprovação do Programa Municipal de Práticas Integrativas e Complementares em Saúde (PMPICs) por meio da Lei Municipal no 72/2013 que atende termos da Política Federal de Práticas Integrativas e Complementares, entre elas, a meditação baseada em mindfulness. ${ }^{24-26}$

Percebeu-se que enfermeiros assistenciais que incorporam a prática de mindfulness vivenciam um melhor bem-estar geral, menos estresse, diminuem os níveis de ansiedade e sintomas de depressão por meio de intervenções que duraram de quatro semanas até oito semanas. Ressalta-se que os impactos positivos da técnica podem ser observados tanto para o profissional quanto para a organização. ${ }^{28}$

Ao ensinar o praticante a vivenciar as experiências do presente com abertura e desprovido de julgamentos ou classificações automáticas, cria-se um processo interno de 
experiências e aquisição de insights sobre as formas de pensamento e atitudes em determinadas situações; insights são importantes nos problemas que surgem no mundo da liderança e eles não podem ser ensinados, mas estão presentes nas experiências do cotidiano. ${ }^{27}$ A flexibilidade às experiências do cotidiano é importante para que enfermeiros e líderes experientes evitem realizar ações e decisões sem a atenção necessária na situação.

A melhora metacognitiva resulta em menor distração no ambiente clínico, permitindo a identificação de mudanças súbitas na condição de saúde dos clientes e melhora no desempenho, podendo reduzir os erros durante os procedimentos de enfermagem, contribuindo assim, para a segurança do paciente. Na clínica os procedimentos hospitalares são melhorados quando os líderes criam um ambiente mais positivo para a prática de enfermagem com redução significativa de eventos adversos como infecção hospitalar, pneumonia, complicações pós-operatórias, tempo de internação, dentre outros. ${ }^{28}$

Além disso, a comunicação com usuários e liderados torna-se mais efetiva, evitando situações de crise. Por isso, a atenção plena tem sido utilizada na clínica médica para diminuir erros médicos, melhorar a relação interpessoal, prevenir o burnout e doenças relacionadas ao estresse. $\mathrm{Na}$ enfermagem, pode ajudar na construção de habilidades que permitirão o engajamento com o usuário de forma significativa, focada e sem pressa.

Ao adotar um referencial terapêutico baseado em mindfulness, a enfermagem ocupa a posição ideal para utilizar, indicar e orientar essas intervenções para os seus clientes, pois esses programas já foram implementados e testados nas mais diversas populações. Ao participar de programas baseados em mindfulness, pode-se adquirir conhecimentos e exercícios básicos que podem ser disseminados para os seus clientes. ${ }^{11}$ Por exemplo, desenvolver técnicas de respiração, aceitação e abertura sem julgamento dos pensamentos 
sobre a experiência das dores e dos medos em usuários vivenciando o processo de adoecimento.

Este estudo sugere que a implementação de práticas específicas direcionadas a aliviar o sofrimento dos indivíduos poderiam ser elegidas na composição do cuidado fundamental de enfermagem. A intervenção sugerida aqui inclui o fornecimento de educação do cliente para reconhecer a resposta do corpo ao estresse e, em seguida, fornecer recursos para a redução de estresse baseada em mindfulness, interrompendo o impacto negativo da resposta ao estresse prolongado. Essas técnicas disponibilizam ferramentas para os indivíduos lidarem com a experiência emocional e o impacto fisiológico da sua doença, trauma ou hospitalização.

É importante enfatizar que não existe uma diferenciação da prática em si de mindfulness, esse diferencial dependerá da natureza da profissão de quem utiliza a prática. No caso dos enfermeiros, por serem os profissionais da saúde que passam a maior parte do tempo interagindo e cuidando de indivíduos em situação de adoecimento e busca por melhores parâmetros de saúde e bem estar, os sentimentos de compaixão, empatia e controle do estresse proporcionados pela prática de mindfulness são benéficos tanto para o profissional quanto para o usuário.

Enfermeiros têm uma oportunidade única de trabalhar com indivíduos e ajudá-los a utilizar práticas integrativas em conjunto com as práticas médicas tradicionais ocidentais. Devendo ser capacitados a pensar de forma integral sobre os cuidados ministrados e desenvolver intervenções que contemplem a complexidade da experiência humana no processo saúde-doença. Enfermeiros são também educadores e têm oportunidade de promover melhores resultados em saúde, ampliando a qualidade de vida a partir de técnicas integrativas. 
O potencial da prática de mindfulness pela enfermagem envolve o tratar e prevenir problemas relacionados com o estresse e promover estratégias de enfrentamento consideradas mais assertivas. Recomenda-se o investimento no avanço das pesquisas que avaliem este tipo de intervenção na equipe de enfermagem. Outra recomendação que atravessa os estudos da área é a necessidade de que os resultados dessas intervenções devem ser acompanhados constantemente, possibilitando o aprimoramento das técnicas e das formas de avaliação, em busca das melhores evidências científicas. ${ }^{29}$

Um dos limites apontados para o estudo consiste no delineamento de pesquisa ideal, pois diferentemente das intervenções farmacológicas, as intervenções baseadas em mindfulness requerem protocolos mais longos que exigem horas de treino e engajamento na prática. Por isso, sugere-se que o melhor delineamento seria o de estudos de coorte comparativo de longo prazo, ou seja, longitudinais com a comparação entre três grupos, por exemplo, de tratamento como de costume, de controle ativo e de intervenção. ${ }^{20}$

Também as intervenções promovidas em caráter extensionista, ou seja, não necessariamente delimitadas enquanto uma investigação científica ou com propósitos de pesquisa, podem ser acompanhadas no longo prazo, contribuindo para delineamentos de programas com foco tanto em usuários e familiares dos serviços de saúde, como dos próprios profissionais de enfermagem. ${ }^{29}$

A veiculação desses programas, bem como a exploração de suas características, seus limites e potencialidades, pode ser útil na composição de práticas cada vez mais alinhadas às necessidades de cada serviço e de cada grupo, dentro da proposta geral que orienta essas intervenções. Considerar a cultura de referência dos usuários e potenciais praticantes e as condições organizacionais dos sistemas de saúde para a execução dessas propostas parece ser um aspecto que pode ser melhor discutido nos estudos vindouros, o que pode também fomentar uma maior representatividade das publicações brasileiras. ${ }^{29}$ 
A complexidade da formação e do ambiente de trabalho da enfermagem geram diversas demandas, exigindo decisões rápidas e éticas, criatividade, habilidades interpessoais e compaixão. Este estudo apresenta uma alternativa para lidar com o estresse e ansiedade característicos dessa população e de diversos usuários que necessitam de atenção de saúde.

Com esta reflexão, fomenta-se a discussão de um nova temática para a enfermagem enquanto dispositivo potente para a qualificação da assistência e da formação no contexto brasileiro, baseando-se no conhecimento produzido sobre mindfulness nas áreas da saúde e educação. Desafia-se os enfermeiros a buscarem novas abordagens que melhorem sua habilidade de provocar mudanças e aumente sua capacidade de resiliência e prestação de cuidados enquanto sujeitos e profissionais em ambientes potencialmente estressores.

\section{Considerações finais}

As reflexões apontam para diversas potencialidades na prática do mindfulness tanto no autocuidado das enfermeiras quanto no cuidado aos usuários e familiares. Os benefícios decorrentes da prática da atenção plena podem ser observados em níveis fisiológicos, psicológicos e sociais, sustentando a ampliação do interesse científico acerca dessas intervenções. Os produtos resultantes da prática baseada em mindfulness podem trazer benefícios para o manejo do estresse cotidiano, na construção de habilidades para liderar e resolver conflitos, na compaixão para cuidar do outro, no relacionamento empático, dentre outros.

Os limites para a utilização de intervenções baseadas em mindfulness vão desde a complexidade do delineamento de pesquisa que contemple a complexidade das intervenções, passando pelas particularidades individuais de cada profissional até as barreiras do próprio sistema de saúde. Considerando os benefícios já relatados, pode-se 
afirmar que mais pesquisas na área ainda trarão avanços no futuro, com destaque para o papel da enfermagem enquanto importante protagonista nesta construção.

Embora o estudo tenha alcançado seu objetivo, existe limitação no que concerne às pesquisas dos benefícios do mindfulness no contexto brasileiro, essas ainda são escassas, o que foi um obstáculo. Mas por outro lado, mostrar dados de pesquisas experimentais e de campo evidenciam a necessidade imediata de desenvolver estudos que abordem os benefícios e a relação enfermagem e mindfulness. Segundo, existe uma limitação de estudos que abordem mindfulness no campo da enfermagem o que dificulta a avaliação dos níveis de evidência necessários para oferecer uma proposição de ação mais estruturada para a enfermagem.

A sumarização das evidências científicas nesta reflexão pode contribuir para o desenvolvimento de novas intervenções e pesquisas que tomem por base o conhecimento já produzido e aqui sistematizado de modo reflexivo. Sensibilizando, assim, a enfermagem sobre os potenciais benefícios que essa prática pode trazer tanto para profissionais quanto para usuários.

\section{Referências}

1. Brown KW, Ryan RM, Creswell JD. Mindfulness: theoretical foundations and evidence for its salutary effects. Psychol Inq [Internet]. 2007 [acesso em 2017 jul 04];18(4):211-37. Disponível em: http://citeseerx.ist.psu.edu/viewdoc/download?doi=10.1.1.458.9330\&rep=rep1\&type=pdf

2. Kabat-Zinn J. Full catastrophe living: using the wisdom of your body and mind to face stress, pain, and illness. New York: Bantam Books; 2013.

3. Nilsson H, Kazemi A. Mindfulness therapies and assessment scales: A brief review. Int J Psychol Stud [Internet]. 2016 [acesso em 2017 jul 10];8(1):11-9. Disponível em: http://www.ccsenet.org/journal/index.php/ijps/article/view/55744

4. Grant S, Colaiaco B, Motala A, Shanman R, Booth M, Sorbero M, et al. Mindfulness-based relapse prevention for substance use disorders: a systematic review and meta-analysis. J Addict Med [Internet]. 2017 [acesso em 2017 out 30];11(5):386-96. Disponível em: https://www.ncbi.nlm.nih.gov/pmc/articles/PMC5636047/pdf/adm-11-386.pdf 
5. Lebuda I, Zabelina DL, Karwowski M. Mind full of ideas: a meta-analysis of the mindfulnesscreativity link. Pers Individ Dif [Internet]. 2016 [acesso em 2017 jul 10];93:22-6. Disponível em: http://www.sciencedirect.com/science/article/pii/S0191886915006133

6. Westbrook C, Creswell JD, Tabibnia G, Julson E, Kober H, Tindle HA. Mindful attention reduces neural and self-reported cue-induced craving in smokers. Soc Cogn Affect Neurosci [Internet]. 2013 [acesso em 2017 jul 10];8(1):73-84. Disponível em: https://www.ncbi.nlm.nih.gov/pmc/articles/PMC3541484/pdf/nsr076.pdf

7. Tang YY, Lu Q, Feng H, Tang R, Posner MI. Short-term meditation increases blood flow in anterior cingulate cortex and insula. Front Psychol [Internet]. 2015 [acesso em 2017 jul 10];6:212. Disponível em: https://www.ncbi.nlm.nih.gov/pmc/articles/PMC4341506/

8. Fox KCR, Nijeboer S, Dixon ML, Floman JL, Ellamil M, Rumak SP, et al. Is meditation associated with altered brain structure? A systematic review and meta-analysis of morphometric neuroimaging in meditation practitioners. Neurosci Biobehav Rev [Internet]. 2014 [acesso em 2017 jul 12];43:48-73. Disponível em: http://www.sciencedirect.com/science/article/pii/S0149763414000724

9. Singleton O, Hölzel BK, Vangel M, Brach N, Carmody J, Lazar SW. Change in brainstem gray matter concentration following a mindfulness-based intervention is correlated with improvement in psychological well-being. Front Hum Neurosci [Internet]. 2014 [acesso em 2017 jul 12];8:33. Disponível em: https://www.ncbi.nlm.nih.gov/pmc/articles/PMC3927233/pdf/fnhum-08-00033.pdf

10. Stahl JE, Dossett ML, LaJoie AS, Denninger JW, Mehta DH, Goldman R, et al. Relaxation response and resiliency training and its effect on healthcare resource utilization. PLos ONE [Internet]. 2015 [acesso em 2017 jul 13];10(10):e0140212. Disponível em: http://journals.plos.org/plosone/article/file?id=10.1371/journal.pone.0140212\&type=printable

11. Williams H, Simmons LA, Tanabe P. Mindfulness-based stress reduction in advanced nursing practice: a nonpharmacologic approach to health promotion, chronic disease management, and symptom control. J Holist Nurs [Internet]. 2015 [acesso em 2017 jul 13];33(3):247-59. Disponível em: http://journals.sagepub.com/doi/pdf/10.1177/0898010115569349

12. Goldin PR, Gross JJ. Effects of mindfulness-based stress reduction (MBSR) one motion regulation in social anxiety disorder. Emotion [Internet]. 2010 [acesso em 2017 Jul 13];10(1):83-91. Disponível em: http://psycnet.apa.org/record/2010-01983-016

13. Bowen S, Witkiewitz K, Clifasefi SL, Grow J, Chawla N, Hsu SH, et al. Relative efficacy of mindfulness-based relapse prevention, standard relapse prevention, and treatment as usual for substance use disorders: a randomized clinical trial. JAMA Psychiatry [Internet]. 2014 [acesso em 2017 jul 13];71(5):547-56. Disponível em: https://jamanetwork.com/journals/jamapsychiatry/fullarticle/1839290 
14. Segal ZV, Walsh KM. Mindfulness based cognitive therapy for residual depressive symptoms and relapse prophylaxis. Curr Opin Psychiatr [Internet]. 2016 [acesso em 2017 jul 04];29(1):7-12. Disponível em: https://www.ncbi.nlm.nih.gov/pmc/articles/PMC4706736/pdf/nihms747542.pdf

15. Gherardi-Donato ECS, Cardoso L, Teixeira CAB, Pereira SS, Reisdorfer E. Associação entre depressão e estresse laboral em profissionais de

enfermagem de nível médio. Rev Latinoam Enferm [Internet]. 2015 [acesso em 2017 jul 06];23(4):73340. Disponível em: http://www.scielo.br/pdf/rlae/v23n4/pt_0104-1169-rlae-23-04-00733.pdf

16. Lopes SA, Vannucchi BP, Demarzo M, Cunha AGJ, Nunes MPT. Effectiveness of a mindfulness-based intervention in the management of musculoskeletal pain in nursing workers. Pain Manag Nurs [Internet]. 2018 [acesso em 2018 Nov 22];20(1):32-8. Disponível em: https://www.sciencedirect.com/science/article/abs/pii/S1524904217304216

17. Weng HY, Fox AS, Hessenthaler HC, Stodola DE, Davidson RJ. The role of compassion in altruistic helping and punishment behavior. PLos ONE [Internet]. 2015 [acesso em 2017 jul 06];10(12):e0143794. Disponível em:

http://journals.plos.org/plosone/article?id=10.1371/journal.pone.0143794

18. Ratanasiripong P, Park JF, Ratanasiripong N, Kathalae D. Stress and anxiety management in nursing students: biofeedback and mindfulness meditation. J Nurs Educ [Internet]. 2015 [acesso em 2017 jul 02];54(9):520-4. Disponível em: https://www.healio.com/nursing/journals/jne/2015-9-549/\%7Ba09587eb-bb22-49ca-b637-314405f196e3\%7D/stress-and-anxiety-management-in-nursingstudents-biofeedback-and-mindfulness-meditation - divReadThis

19. Song Y, Lindquist R. Effects of mindfulness-based stress reduction on depression, anxiety, stress and mindfulness in Korean nursing students. Nurse Educ Today [Internet]. 2015 [acesso em 2017 jul 02];35(1):86-90. Disponível em: http://www.sciencedirect.com/science/article/pii/S0260691714002226

20. The Telegraph. Nurses 'lacking ability and compassion', warns NHS Future Forum. London; 2012 [acesso em 2017 abr 02]. Disponível em: http://www.telegraph.co.uk/news/health/news/9003490/Nurses-lacking-ability-and-compassionwarns-NHS-Future-Forum.html

21. Buchholz L. Exploring the promise of mindfulness as medicine. JAMA [Internet]. 2015 [acesso em 2017 jul 02];314(13):1327-9. Disponível em: https://jamanetwork.com/journals/jama/articleabstract/2449182? redirect=true

22. Sanko J, Mckay M, Rogers S. Exploring the impact of mindfulness meditation training in prelicensure and post graduate nurses. Nurse Educ Today [Internet]. 2016 [acesso em 2017 jul 02];45:142-7. Disponível em: http://www.sciencedirect.com/science/article/pii/S0260691716301265 
23. Guillaumie L, Boiral O, Champagne J. A mixed-methods systematic review of the effects of mindfulness on nurses. J Adv Nurs [Internet]. 2016 [acesso em 2017 jul 01];73(5):1017-34. Disponível em: http://onlinelibrary.wiley.com/doi/10.1111/jan.13176/epdf

24. Ministério da Saúde (BR). Gabinete do Ministro. Portaria MS/GM n. 971, de 3 de maio de 2006. Aprova a Política Nacional de Práticas Integrativas e Complementares (PNPIC) no Sistema Único de Saúde [Internet]. 2006 [acesso em 2018 nov 22]. Disponível em: http://bvsms.saude.gov.br/bvs/saudelegis/gm/2006/prt0971_03_05_2006.html

25. Ministério da Saúde (BR). Departamento de Atenção Básica (DAB). Portal Saúde. Legislação. Práticas Integrativas e Ccomplementares [Internet]. 2017 [acesso em 2018 nov 22]. Disponível em: http://dab.saude.gov.br/portaldab/biblioteca.php?conteudo=legislacoes/pnpics

26. Ministério da Saúde (BR). Gabinete do Ministro. Portaria n. 702, de 21 de março de 2018. Altera a Portaria de Consolidação n. 2/GM/MS, de 28 de setembro de 2017, para incluir novas práticas na Política Nacional de Práticas Integrativas e Complementares - PNPIC0 [Internet]. 2018 [acesso em 2018 nov 22]. Disponível em: http://bvsms.saude.gov.br/bvs/saudelegis/gm/2018/prt0702_22_03_2018.html

27. Kavanagh KT, Cimiotti JP, Abusalem S, Coty MB. Moving healthcare quality forward with nursing-sensitive value-based purchasing. J Nurs Scholarsh [Internet]. 2012 [acesso em 2017 jul 02];44(4):385-95. Disponível em: http://onlinelibrary.wiley.com/doi/10.1111/j.15475069.2012.01469.x/epdf

28. Howland LC, Bauer-Wu S. The mindful nurse. Am Nurs Today [Internet]. 2015 [acesso em 2017 abr 04];10(9). Disponível em: https://www.americannursetoday.com/mindful-nurse/

29. White L. Mindfulness in nursing: an evolutionary concept analysis. J Adv Nurs [Internet]. 2014 [acesso em 2017 ago 02];70(2):282-94. Disponível em: http://onlinelibrary.wiley.com/doi/10.1111/jan.12182/abstract

\section{Fomento}

Conselho Nacional de Desenvolvimento Científico e Tecnológico (CNPq) Universal MCTI/CNPq 01/2016 Processo número 424062/2016-0

\section{Autor correspondente}

\section{Edilaine Cristina da Silva Gherardi-Donato}

E-mail: gherardidonato@gmail.com

Endereço: Escola de Enfermagem de Ribeirão Preto USP

Av. Bandeirantes, 3900. Monte Alegre. Ribeirão Preto-SP, Brasil.

CEP: $14.040-902$ 


\section{Contribuições de Autoria}

\section{1 - Edilaine Cristina da Silva Gherardi-Donato}

oncepção e planejamento do artigo, obtenção dos dados, redação e revisão crítica.

\section{2 - Maria Neyrian de Fátima Fernandes}

Concepção e planejamento do artigo, obtenção dos dados, redação e revisão crítica.

3 - Fabio Scorsolini-Comin

Redação e revisão crítica.

4 - Ana Carolina Guidorizzi Zanetti

Obtenção dos dados, redação e revisão crítica.

\section{Como citar este artigo}

Gherardi-Donato ECS, Fernandes MNF, Scorsolini-Comin F, Zanetti ACG. Mindfulness: reflexão sobre limites e potencialidades para a assistência de enfermagem. Rev. Enferm. UFSM. 2019 [Acesso em: Anos Mês Dia];vol.9, e52: P1-21. DOI:https://doi.org/10.5902/2179769233058 